\title{
EXCITED-STATE EQUILIBRATION AND THE FLUORESCENCE-ABSORPTION RATIO
}

\author{
R.S. KNox \\ Department of Physics and Astronomy and Rochester Theory Center \\ for Optical Sciences and Engineering, University of Rochester \\ Rochester, NY 14627-0171 USA
}

\begin{abstract}
In any complex system at temperature $T$ the absorption cross-section and fluorescent power at a given photon energy are connected by a simple relation if the system is in thermal equilibrium while occupying one particular electronic excited state. Although this situation is impossible in principle because of finite excited-state lifetimes, it is often approximated to the extent that the simple relation, which is expressed as a linear function of energy with slope $-1 / k_{\mathrm{B}} T$, holds in a variety of cases. (The usual symbols for Boltzmann's constant and absolute temperature are used.) Observed deviations are of two principal kinds: a slope characteristic of some temperature $T^{*}$ other than ambient, and departures from a single pure straight line. The latter may include seemingly random variations and in some cases multiple regions of straight-line behavior. We have recently introduced an effective temperature $T^{*}(E)$, derived from the actual local slope of the putative straight line at energy $E$, which turns out to be a very sensitive detector of deviations from the ideal and, we believe, from equilibrium in the excited state. Plots of $T^{*}(E)$ display a variety of features. An anomaly in the $T^{*}(E)$ spectrum of chlorophyll $a$ can be analyzed on this model, indicating a second weakly fluorescent state about $70 \mathrm{meV}$ below the well-known $Q_{y}$ band. The cases of chlorophyll and many others are included in a selective review of applications of the universal relation to fluorescent systems.
\end{abstract}

PACS numbers: $42.50 . \mathrm{Md}, 33.70 . \mathrm{Jg}, 32.50 . \mathrm{d}, 31.70 . \mathrm{Hq}$

\section{Introduction}

\subsection{Meaning of equilibration in an excited state}

Pringsheim was apparently the first, in 1913 , to introduce a thermal characterization of non-thermal radiation [1]. In 1918 Kennard [2] found a simple relationship between fluorescence and absorption spectra and later revised the derivation on the basis of the emerging quantum theory [3]. The relationship, which will be found below as Eq. (7), was brought into its modern form by Stepanov [4] and Neporent [5]. Let us first consider the physics involved. 
Excited states that produce visible radiation can be prepared thermally, but this happens at a very small rate. Indeed, since we expect the upward transition rate to be $\exp \left(-\Delta E / k_{\mathrm{B}} T\right)$ times the radiative rate in fluorescence, we shall wait, on average, $1.9 \times 10^{14}$ years to perceive a red $(\Delta E=1.8 \mathrm{eV})$ thermal photon at room temperature. This is a consequence of the exceptionally small high-energy tail (the Wien limit) of the Planck distribution at $T=295 \mathrm{~K}$. When a fluorescent state is excited directly, this tail is effectively amplified. The formalism of Kennard and Stepanov is essentially based upon a shift of the zero of energy in the Boltzmann factor from the ground electronic state to some principal excited electronic state. After the preparation of that state the system is assumed quickly to equilibrate with its thermal bath, sufficiently quickly that all of the observed emission originates from the so-equilibrated states.

We can expect deviations from the ideal relation to occur simply because a given system may have inadequate time to reach the assumed excited-state equilibration before most of the radiation is to be emitted. Two factors reducing this time are the radiative process itself, which might be too fast, and the dissipation (or acquisition) of thermal energy, which might be too slow. The rates of all relevant vibrational, librational, conformational, and solvation processes must be at least a factor of 10 or 100 larger than the radiative rate.

\subsection{A general expression for the emission-absorption relationship}

We consider a system with two important electronic states, the ground state at $E_{0}=0$ and the excited state at $E_{1}$. Associated with these are bath states whose energies will be called $w$ and $E_{1}+w^{\prime}$ and which are assumed to comprise continua with densities $g(w)$ and $g^{\prime}\left(w^{\prime}\right)$, respectively. For absorption, the initial (ground) state is assumed to be in equilibrium with the bath, so that the relative probability of occupation of states at $w$ is $\exp \left(-w / k_{\mathrm{B}} T\right)$. Similarly, when the assumption of excited-state equilibration is made, the relative probability of occupation of states associated with the excited electronic state at energy $E=E_{1}+w^{\prime}$ is assumed to be $\exp \left(-w^{\prime} / k_{\mathrm{B}} T\right)$. In what follows we first assume some average excited-state probability distribution $p\left(w^{\prime}\right)$. More generally, it may be time-dependent, $p\left(w^{\prime}, t\right)$.

The absorption spectrum may be expressed with considerable generality as

$$
\sigma(E)=Z^{-1} \int g(w) B(w, E) \exp \left(-w / k_{\mathrm{B}} T\right) \mathrm{d} w,
$$

where $B(w, E)$ is the Einstein $B$ coefficient in appropriate units for transitions from states at energy $w$ to electronically excited states at energy $w+E$, and $Z$ is the partition function

$$
Z=\int g(w) \exp \left(-w / k_{\mathrm{B}} T\right) \mathrm{d} w
$$

In certain well-defined situations the "hot" fluorescence spectrum may be regarded as having a time-dependent shape function while relaxation occurs (see, for example, Mukamel and Rupasov [6]). In our context this means that the emission rate, in quanta per second per unit bandwidth, may be written

$$
f(E, t)=Z^{\prime-1} \int g^{\prime}\left(w^{\prime}\right) A\left(w^{\prime}, E\right) p\left(w^{\prime}, t\right) \mathrm{d} w,
$$


where we make use of the probability function $p$ introduced above and

$$
Z^{\prime}(t)=\int g^{\prime}\left(w^{\prime}\right) p\left(w^{\prime}, t\right) \mathrm{d} w^{\prime}
$$

The genesis of the emission-absorption relationship that we seek is the Einstein $A-B$ relation, which in our context refers to the groups of states in the vicinity of $w$ and $w^{\prime}$ that are participating in transitions at photon energy $E$,

$$
g^{\prime}\left(w^{\prime}\right) A\left(w^{\prime}, E\right) \mathrm{d} w^{\prime}=\frac{8 \pi E^{3}}{h^{3} c^{3}} g(w) B(w, E) \mathrm{d} w .
$$

The relation we are developing is usually presented in terms of a luminescent power spectrum, $I(E, t)=E f(E, t)$, and the $B$ coefficient can be related to the absorption cross-section by $\sigma(E)=E B(E) / c$. Using these conventions and combining Eqs. (1), (3), and (5), we obtain

$$
\frac{h^{3} c^{2}}{8 \pi} \frac{I(E, t)}{E^{3} \sigma(E)}=\frac{Z \int g(w) B(w, E) p\left(w^{\prime}, t\right) \mathrm{d} w}{Z^{\prime}(t) \int g(w) B(w, E) \exp \left(-w / k_{\mathrm{B}} T\right) \mathrm{d} w} .
$$

The medium index of refraction has no substantial consequence for our application of this equation and has therefore been ignored.

\subsection{The special case of Kennard and Stepanov}

In the absence of very specific knowledge of $g(w)$ and $B(w, E)$, the combination produced in Eq. (6) appears to have little use. However, if the steady-state emission is considered and a time-averaged $p\left(w^{\prime}, t\right)$ is approximated by the Boltzmann distribution, the unknown functions cancel completely, leaving

$$
\begin{aligned}
F(E) & =\ln \left[\frac{h^{3} c^{2}}{8 \pi} \frac{I(E)}{E^{3} \sigma(E)}\right] \\
& =\ln \left[\frac{\left.Z \int g(w) B(w, E) \exp \left(-w-E+E_{1}\right) / k_{\mathrm{B}} T\right) \mathrm{d} w}{Z^{\prime} \int g(w) B(w, E) \exp \left(-w / k_{\mathrm{B}} T\right) \mathrm{d} w}\right] \\
& =\ln \left(\frac{Z}{Z^{\prime}}\right)+\frac{E_{1}-E}{k_{\mathrm{B}} T} .
\end{aligned}
$$

(We have used the fact that $w+E=E_{1}+w^{\prime}$ and therefore $w^{\prime}=w+$ $E-E_{1}$. ) Equation (7) is the Kennard-Stepanov (KS) "universal relation". It makes the interesting prediction that the function $F(E)$, which is readily obtained from experiment, is linear in $E$. The first term in the last line of Eq. (7), and $E_{1}$, will vary with the system under consideration and may be temperature dependent, but are independent of $E$.

A more detailed version of this derivation was recently given by Laible et al. [7], who studied the connection between Eq. (7) and the Förster theory of excitation transfer. That theory rests on the same assumptions about the equilibration of the excited state. 


\subsection{History and scope of the relation}

After the early work by Pringsheim and Kennard, little appears on the subject until Stepanov's revival of the work. Attention in the 1930s had turned largely to fluorescence polarization (Perrin [8], Jabłonski [9]) but the thermodynamic aspect did surface in a lively debate over the possibility of anti-Stokes luminescence in the face of the First Law [10-13].

In the twenty years following the work of Stepanov and Neporent dozens of organic compounds as vapors and in solution were subjected to analyses to test the emission-absorption relationship. Some of this activity will be reviewed in the following section. The relationship has also been used in connection with semiconductor spectra, where it is known as the "reciprocity" relation $[14,15]$.

Section 2 contains a survey of analyses that have been made in the context of determining an effective bath temperature from Eq. (7). This has been the thrust of almost all investigations since 1957. In Sec. 3 we describe an alternative procedure in which a more detailed look at the energy dependence of $F(E)$ is taken by introducing a spectral temperature $T^{*}(E)$. Section 4 discusses attempts to understand $T^{*}(E)$ and Sec. 5 describes a case study, that of chlorophyll $a$. A brief look at the time-resolved case is taken in Sec. 6, and we summarize in Sec. 7.

\section{Experimental tests of the fluorescence-absorption relation}

It is important to keep in mind that the relation under study, Eq. (7), speaks about absorption and emission at the same photon energy. Therefore, as a practical matter, only a limited range of energies can be explored. The region in which data are normally "best" for the analysis is the Stokes region (between the peak emission and absorption). Data outside this region must be corrected carefully. For example, there may be reabsorption and secondary emission in the case of the emission spectrum and emission and scattering artifacts in the case of the absorption spectrum. The theorist must be aware of possible limitations on data that may have been obtained for purposes other than a rigorous test of the KS relation. In this survey we assume that all data have been conditioned to an appropriate level of accuracy.

\subsection{Historical examples of apparently linear $F(E)$}

Figure 1 is a typical plot obtained by applying the first line of Eq. (7) to fluorescence and absorption data. The example is adapted from published spectra of trypaflavin in methanol [16] and shows a reasonably linear $F(E)$ as the ratio of $I(E) / E^{3} \sigma(E)$ ranges over three orders of magnitude. The slope implies an effective bath temperature $T^{*}=325 \mathrm{~K}$.

Among the many cases in which the KS relation is found experimentally to produce a linear function of $E$, one may distinguish three main classes: those in which the relation is found to be obeyed to within reasonable error limits; those in which the apparent temperature, which we will call $T^{*}$, is higher than the ambient $T$ by an appreciable amount, say 10 to $40 \%$; and those in which the value of $T^{*}$ falls within the first two cases but also depends strongly on excitation wavelength. 


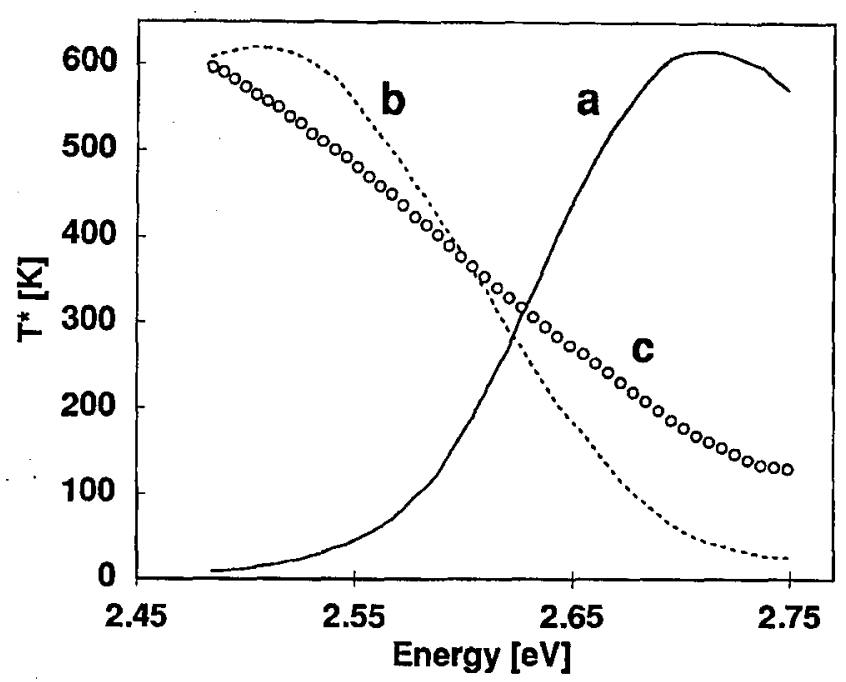

Fig. 1. Example of the traditional application of Kennard-Stepanov theory. Shown are (a) absorption and (b) emission spectra of $0.3 \mathrm{mM}$ trypaflavine in methanol at $293 \mathrm{~K}$ (adapted from Ketskemety et al. [16]). The experimentally derived KS function $F(E)$, computed by applying the first line of Eq. (7), is plotted with circles (c). The effective temperature deduced from the slope of $F(E)$ by the authors was $325 \mathrm{~K}$.

TABLE I

Examples of Kennard-Stepanov parametric temperatures lying within about $1 \%$ of ambient temperature.

\begin{tabular}{l|l|c|c|c}
\hline \hline \multicolumn{1}{c|}{ Solute } & \multicolumn{1}{|c|}{ Solvent } & $\begin{array}{c}T \text { (ambient) } \\
{[\mathrm{K}]}\end{array}$ & $\begin{array}{c}T^{*} \\
{[\mathrm{~K}]}\end{array}$ & Ref. \\
\hline allophycocyanin & K phosphate buffer & 295 & 294 & {$[17]$} \\
3-aminophthalimide & propyl alcohol & 293 & 290 & {$[18]$} \\
chlorophyll $a$ & carbon disulfide & 296 & 298 & {$[19]$} \\
9,10-diphenylanthracene & cyclohexene & 293 & 292 & {$[20]$} \\
fluorescein & glycerin & 298 & 299 & {$[21]$} \\
rhodamine 6G & water & 293 & 295 & {$[22]$} \\
trans-stilbene & dioxane & 298 & 300 & {$[23]$}
\end{tabular}

A fourth and much smaller class could be defined in which $T^{*}$ is anomalous, being well outside the range $0.9 T<T^{*}<1.4 T$. This class will not be discussed here.

Table I contains a selection of solutes/solvents (or vapors) that appear to approach Kennard-Stepanov ideal conditions [17-23]. We do not show error estimates, because the requisite information is not available from most of these old papers and it was apparently seldom considered necessary to publish known uncertainties. Upon first seeing the result of Eq. (7) that arises from a pair of very 
complex spectra, one can be struck by the large range over which a linear relation holds. Indeed, we have found that this may often have had a mesmerizing effect, making lines seem straighter and the reported temperature closer to ambient than the data imply. This is not felt to be the case for anything reported in Table I, however.

TABLE II

Examples of Kennard-Stepanov parametric temperatures considerably higher than ambient temperature.

\begin{tabular}{l|c|c|c|c|c}
\hline \hline \multicolumn{1}{c|}{ Solute } & Solvent & $\begin{array}{c}T \text { (ambient) } \\
{[\mathrm{K}]}\end{array}$ & $\begin{array}{c}T^{*} \\
{[\mathrm{~K}]}\end{array}$ & $T^{*} / T$ & Ref. \\
\hline 2,5-di(4-biphenylyl) oxazole & PMMA $^{a}$ & 298 & 430 & 1.44 & {$[24]$} \\
3,6-diaminophthalimide & (vapor) & 538 & 724 & 1.35 & {$[25]$} \\
dimethyl-amino-nitro-stilbene & EPA $^{b}$ & 163 & 212 & 1.30 & {$[26]$} \\
erythrosin & ethanol $^{-}$ & 338 & 456 & 1.35 & {$[27]$} \\
flavophosphin & ethanol & 262 & 344 & 1.31 & {$[27]$} \\
perylene & (vapor) & 513 & 556 & 1.08 & {$[25]$} \\
rose bengal & ethanol & 262 & 351 & 1.34 & {$[27]$} \\
trypaflavin & glycerine & 262 & 342 & 1.31 & {$[27]$} \\
trypaflavin & 1-butanol & 293 & 391 & 1.33 & {$[16]$} \\
\hline
\end{tabular}

${ }^{a}$ polymethyl methacrylate, ${ }^{b}$ ether : pentane : ethanol, $5: 5: 2$ by volume

Table II shows a selection of cases [16, 24-27] in which the anticipated linear relation is found, but "incorrect" temperatures are implied by the data. The first reaction to such a finding may be to envision some heating of the local environment by excess photon energy, and some attempts were made [16] to connect this with Jabłoński's ideas about excess excitation energy dissipation [28]. This point of view does not withstand close scrutiny in the KS-relation context (see next subsection). A competing hypothesis to explain the elevated $T^{*}$ is based on inhomogeneous broadening [29].

The third class of linear KS systems is well represented by the sequence of chlorin derivatives whose parametric temperatures are shown in Fig. 2. As the excitation energy is increased, $T^{*}$ increases in a quite regular manner [30]. Such a result might appear to support strongly the idea of local heating by excess energy deposition in the excited state, but it, too, may possibly be explained on the basis of inhomogeneous broadening [29].

A remarkable example of the third class was discovered by Sechkarev and Beger [31]. In adsorbed rhodamine 6G, they observed a periodic variation in $T^{*}$ as a function of excitation energy, which they attributed to a specific bottleneck for vibrational energy transfer. This system should be especially interesting to treat using the alternative method we will outline in Sec. 3 . 


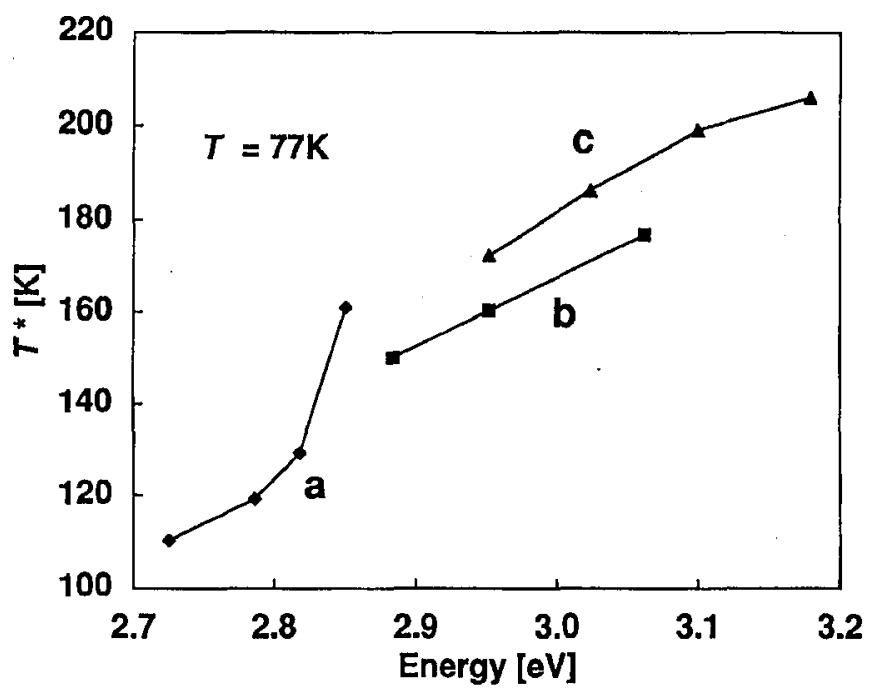

Fig. 2. Excitation-energy dependence of the effective KS temperatures $T^{*}$ for three related chromophores (a) chlorophyll $a$, (b) pheophytin $a$, and (c) chlorin. Solvent was EPA (ether : pentane : ethanol, 5:5:2 by volume) and ambient temperature was $77 \mathrm{~K}$. In each case the absorption maximum corresponding to the fluorescing state was at $665 \pm 3 \mathrm{~nm}$, i.e., within $2 \%$ of $1.86 \mathrm{eV}$ (adapted from Hevesi and Singhal [30]).

\subsection{How are $T^{*}>T$ and an excitation-dependent $T^{*}$ to be understood?}

The question asked here seems not to have been explored since it was discussed and reviewed in 1976 [29]. This is probably due in large part to the development of time-resolved spectroscopy and the concomitant change in research interests based on a well-justified hope that excited-state relaxation processes may be best understood by more specific and detailed studies. Nonetheless, the simplicity of the KS theory and the unusual nature of the related observations continue to be of interest.

Emission characteristic of a molecule at temperature $T^{\prime}>T$ may be called warm fluorescence. The concept is rather tenuous because the excited manifold is expected to be equilibrating toward $T$ during the emission process. But assume that most of the fluorescence does originate during a time in which the molecule is effectively at $T^{\prime}$. Can this be the $T^{*}$ that is seen in a KS analysis? There are reasons to think not. Since the KS relation involves both absorption and emission, there must be two temperatures involved in the $\mathrm{KS}$ expression. When the second temperature is actually introduced [29, 32] a distinct curvature is produced in the formerly linear relation. The correction to the KS theory is rather straightforward on the two-temperature model [29];

$$
F(E)=\ln \left[\frac{I\left(E, T^{\prime}\right)}{E^{3} \sigma(E, T)}\right]=\operatorname{const}\left(T, T^{\prime}\right)-\frac{E}{k_{\mathrm{B}} T^{\prime}}+\ln \left[\frac{\sigma\left(E, T^{\prime}\right)}{\sigma(E, T)}\right] .
$$

The third term on the right introduces the above-mentioned curvature. Equation (8) makes the prediction that a simple "warm" temperature may appear in 
the KS results if the absorption spectrum at temperature $T^{\prime}$ is the same as it is at temperature $T$. This model is therefore open to a fairly simple test. We believe it has not been made.

The effect of inhomogeneous broadening has been considered on the basis of both broad [29] and detailed [33] models. To produce a change in the original KS relation it is not sufficient merely to invoke a distribution of transition energies. One must also specify the rate of excitation transfer among the molecules in the case of static broadening or the rate of fluctuation in the case of dynamic broadening. Should these rates be large, the system would again be in equilibrium and should present the normal KS relation.

Let us assume the case of static disorder with no transfer of excitation between molecules, as in [29]. In this case the KS relation acquires two corrections. The effective KS temperature $T^{*}$ becomes larger

$$
T^{*}=\left[\left(a^{2}+2 b^{2}\right) /\left(a^{2}+b^{2}\right)\right] T,
$$

where $b$ is the width of the inhomogeneous envelope and $a$ is the homogeneous width. Secondly, an excitation-energy-dependent term is added. This explanation appears quite promising, but the theory makes a third prediction, namely, a considerable emission peak shift during a change in excitation energy. To our knowledge, this has not been tested systematically, and has failed to be confirmed in one case, chlorophyll $a$ in solution [34].

\subsection{The case of mixtures of non-interacting chromophores}

Consider two different but otherwise homogeneous solutes $A$ and $B$, each of which separately satisfies the KS relation with

$$
F_{A}(E)=c_{A}-\left(E-E_{A}\right) / k_{\mathrm{B}} T, \quad F_{B}(E)=c_{B}-\left(E-E_{B}\right) / k_{\mathrm{B}} T .
$$

If the solutes do not interchange energy and exist in fractional concentrations $f_{A}$ and $f_{B}$, the overall $\mathrm{KS}$ function is readily found to be

$$
F_{A B}(E)=\ln \left[\frac{f_{A} \sigma_{A} \exp \left(F_{A}\right)+f_{B} \sigma_{B} \exp \left(F_{B}\right)}{f_{A} \sigma_{A}+f_{B} \sigma_{B}}\right] .
$$

The result is shown in Fig. 3 , where we have chosen a simplified set of constants and Gaussian profiles for the absorption bands. One can see on inspection of Eq. (11) that the asymptotic regions occur where the absorption of $B$ is negligible, in which case $F_{A B}=F_{A}$, and where the absorption of $A$ is negligible, in which case $F_{A B}=F_{B}$. The slope of the curve in the region between asymptotes is smaller in magnitude than $1 / k_{\mathrm{B}} T$ and may be reversed in sign if the energy difference $E_{A}-E_{B}$ becomes sufficiently large. Björn and Björn introduced this type of analysis in a study of phycobilisome spectra [35].

This discussion helps to understand the fact that $T^{*}>T$ under inhomogeneous broadening conditions. When many species exist with a spread of energies, many smaller kinks of the type shown in Fig. 3 combine to lower the slope of $F$ over a wide region, thereby inducing a higher effective temperature. 


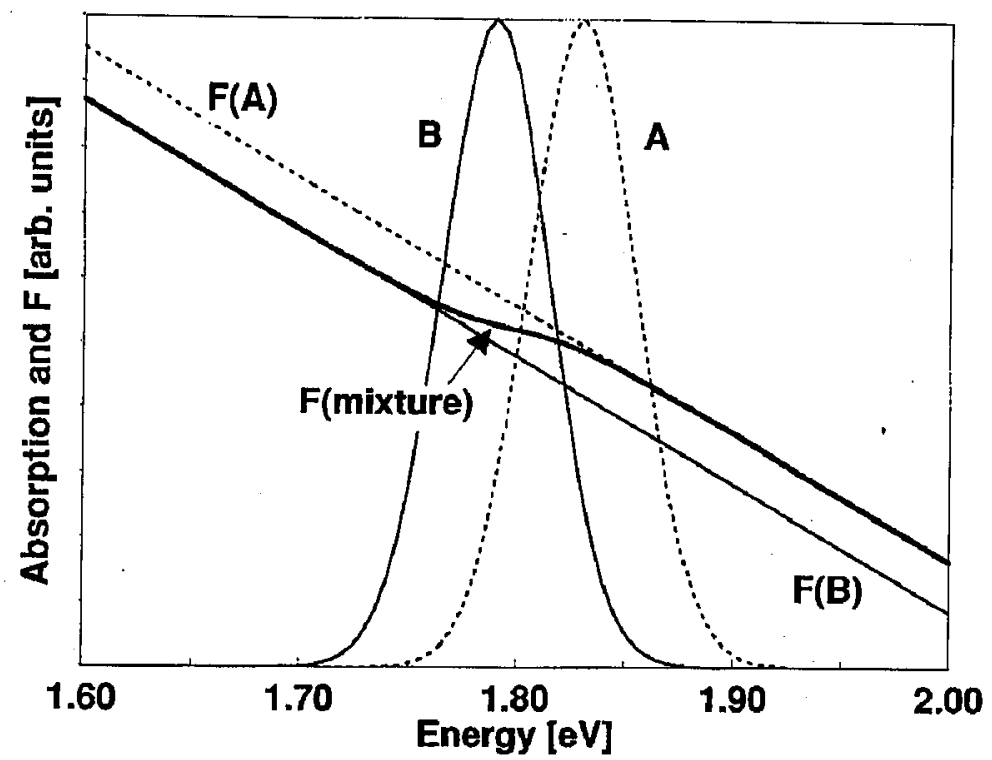

Fig. 3. The KS relation resulting from a non-interacting mixture of two species having different excited-state energies. Fluorescence spectra are not shown. Species A: dotted lines. Species B: light solid lines. The heavy solid line is the function $F(E)$ for the mixture.

\subsection{The case of $\alpha$-phycocyanin}

A set of data on the chromophore $\alpha$-phycocyanin [36], an important photosynthetic pigment, led to the curve shown in Fig. 4. While the sample consisted of a single chromophoric species, the data when subjected to KS analysis [36, 37] strongly indicated more than one species, in the context of the preceding section. We include this example not because it is now well understood, but because it illustrates a point that will be important to the following development. That is, even in a monolithic and homogeneous sample, there may be the equivalent of a mixture of species, in that different electronic manifolds may equilibrate separately without good kinetic contact among them. The basic KS theory assumes only one fluorescing electronic excited state; this is not a limitation provided that all other electronic states are sufficiently well coupled to it, to the extent that they essentially act as bath states.

As proposed tentatively by Sauer [36], the chromophore in alpha-phycocyanin could also represent the case of a single electronic excited state but one that is transformed by a molecular conformation. The two conformation states could easily have different emissive properties, and the kinetics of these states might resemble the kinetics of excitation transfer, either intramolecular or intermolecular. Clearly, in the final analysis, a complete understanding of the KS function for a given system will depend on much corroborative information, but the KS function can itself inform other studies. 


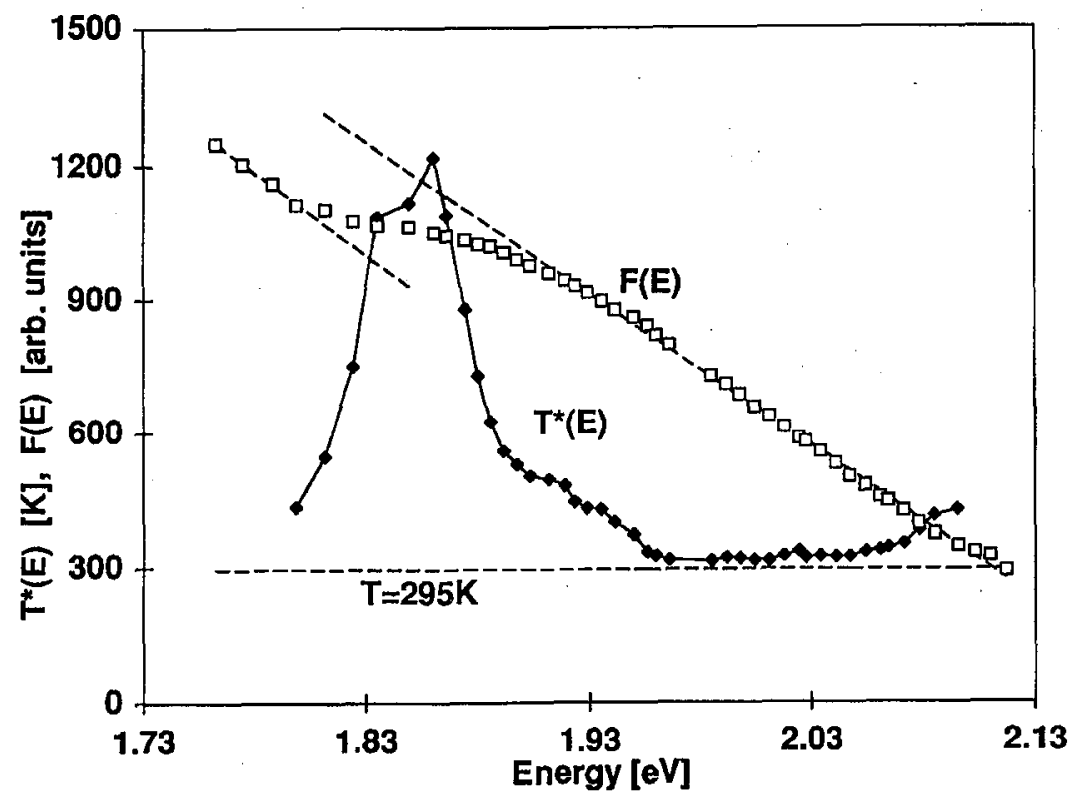

Fig. 4. The KS relation obtained experimentally by Sauer [36] for $\alpha$-phycocyanin. The dashed lines are qualitative indications of the two quasi-linear portions of the function $F(E)$; Eq. (7). $T^{*}(E)$ was calculated from Eq. (12) (after Sawicki and Knox [37]).

\section{An alternative method of data presentation}

\subsection{A careful study of $F$ in Cornell dyes led to $T^{*}(E)$}

In early 1995 , we undertook to compare the value of $T^{*}$ in a sequence of similar dyes in which the fluorescence lifetime differed significantly (measurements by Laible [38]). In the case of oxazine 725 , with a lifetime of $930 \mathrm{ps}$, we found $T^{*}=300 \mathrm{~K}$, while in DDCI $\left(1,1^{\prime}\right.$-diethyl-2, $2^{\prime}$-dicarbocyanine iodide), with a lifetime of $25 \mathrm{ps}$, we found $T^{*}=317 \mathrm{~K}$. The trend was inconclusive, but the study proved interesting in a different aspect: having the complete electronic data set and the knowledge that the data were well corrected, we were able to examine the slope of the $F(E)$ curve in greater detail. When the slope was evaluated locally, and a temperature deduced at every point on the curve, we found the phenomenon shown in Fig. 5. The effective temperature varied considerably even within the Stokes region. The definition of $T^{*}(E)$ used [37] was

$$
T^{*}(E)=-\left[k_{\mathrm{B}} \frac{\mathrm{d} F}{\mathrm{~d} E}\right]^{-1}=-\left[k_{\mathrm{B}} \frac{\mathrm{d}}{\mathrm{d} E} \ln \left(\frac{h^{3} c^{2}}{8 \pi} \frac{I(E)}{E^{3} \sigma(E)}\right)\right]^{-1} .
$$

This spectrally-dependent temperature should be considered only vaguely suggestive of a physical temperature until its variations can be better understood. It is best regarded as merely an extended set of $T^{*}$ parameters characterizing the experimentally-measured $F(E)$ function. In Sec. 4 we describe our attempts to relate $T^{*}(E)$ to relaxation phenomena. 


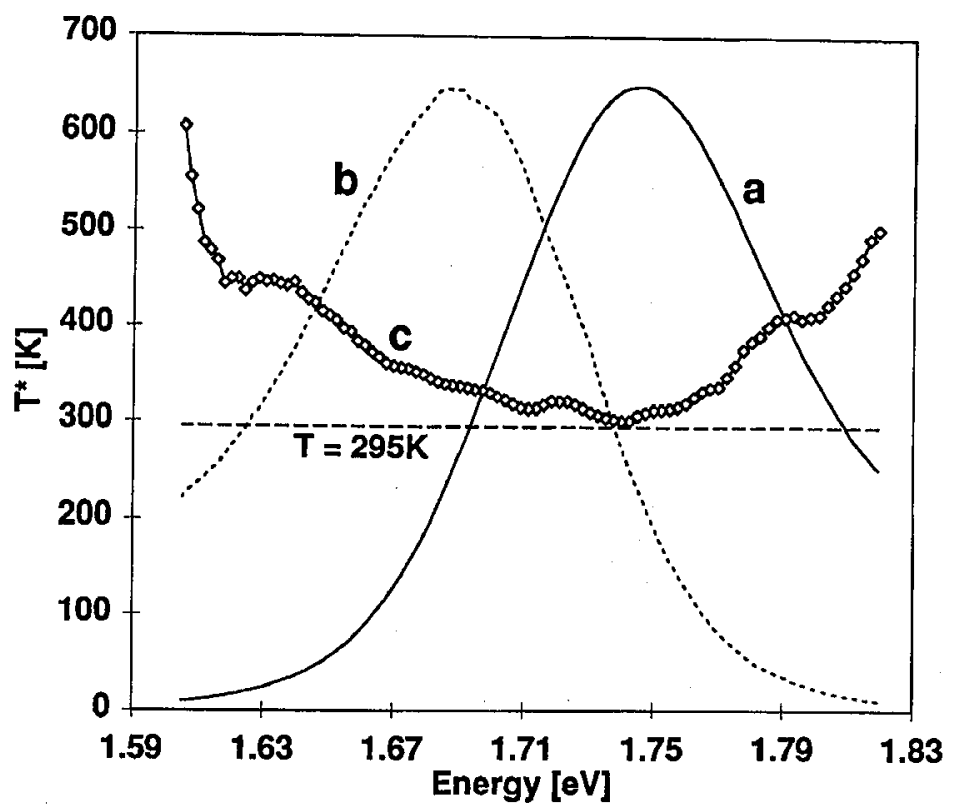

Fig. 5. Spectra of DDCI (1, 1'-diethyl-2,2'-dicarbocyanine iodide) at $295 \mathrm{~K}$ : (a) absorption, (b) emission, and (c) $T^{*}(E)$. Emission and absorption data taken by Laible [39].

When the spectral KS temperature is computed for $\alpha$-phycocyanin, a peak emerges in the "kink" region (Fig. 4). As we have analyzed further data we have found many such peaks, some of which are so divergent as to give a negative effective temperature.

\subsection{Reproducibility of $T^{*}(E)$}

$T^{*}(E)$ is a magnifier of small effects, and in this respect it is similar to the result of derivative spectroscopy. Since many of the unusual features we have seen occur in the region of the absorption and fluorescence tails, impurities may at once be suspected as the cause, but we have not thus far been able to identify any such cases. One would expect that the effect of impurities would be to make features of $T^{*}(E)$ quite variable, especially in spectral position, but most variability seen thus far has been in the strength of peaks.

If emission and absorption spectra in two instances are identical, $T^{*}(E)$ will obviously be reproduced. An interesting case is that of PPV, poly(phenylenevinylene), a polymer of rather heterogeneous local structure. As reported in our original paper [37], similar KS temperature spectra were found when derived from data on several different PPV samples prepared on different days [39], and reasonable error bars could be placed on $T^{*}(E)$ despite considerable differences in the absorption and fluorescence from which it was derived.

Reproducibility of $T^{*}(E)$ within even one set of data is also an issue. The exact shape of $T^{*}(E)$ depends on how many data points are used to establish the derivative. If only three are used, the result is usually very noisy. If the entire 
data set is used, all features vanish and there is only one value of $T^{*}$, the original parametric one. Some judgment is therefore necessary. We have found that no qualitative difference is introduced when different numbers of points are chosen, over a range of 5 to 21 points; 9 points is our standard if the result is not noisy. On the experimental side, a miscalibration of either of the two spectra with respect to the other would clearly introduce error into $T^{*}(E)$. We have verified that any reasonably small shift of the scale of either the absorption or fluorescence introduces no qualitative change in $T^{*}(E)$, and very little quantitative change.

\section{Theoretical approaches to $T^{*}(E)$}

\subsection{Qualitative considerations}

Does $T^{*}(E)$ contain more or less information than the two spectra from which it is constructed? The closer it comes to being constant and equal to ambient, the less information it appears to contain, except for the single fact that the excited state acts as if it were equilibrated. When $T^{*}(E)$ contains structure, that one message is reversed, but clearly the structure has potentially more information. In a sense our procedure projects out the Boltzmann distribution and reveals hot spots in the excited-state manifold. It must have some relation, albeit implicit and complex, to the unknown distribution functions $p\left(w^{\prime}\right)$ and $p\left(w^{\prime}, t\right)$ introduced above.

Although the term "hot spot" is attractive, we must ask whether $T^{*}(E)$ can be regarded as a real temperature in any sense. It might be thought, for example, focusing simply on a small region of the excited manifold, that the group of states at $w^{\prime}$ and within some small band $\Delta w^{\prime}$ have a probability distribution approximated by the function $\exp \left[-w^{\prime} / k_{\mathrm{B}} T^{*}\left(E_{1}+w^{\prime}\right)\right]$. This would be technically incorrect because it assumes that all transitions originate and terminate at the origin $(w=0)$ of the ground state manifold. Thus it would be misleading because the energy $E$ appearing in $T^{*}(E)$ is actually the energy of a set of transitions that originate and terminate in various regions of the ground-state manifold, according to the Franck-Condon factors governing the selected set. From a different perspective: a simple deconvolution will not take us from $T^{*}$ to $p$.

\subsection{The coupled- $N$-manifold model}

A simple approach to the equilibration process in a single species is suggested by the case of energy transfer between different species. In Sec. 2.3 we considered the case of a mixture of species $A$ and $B$ and constructed the mixture's KS function (Eq. (11)). Imagine a single species having two manifolds built on two different excited electronic states, and being such that if the system were confined to either one of these manifolds it would equilibrate very quickly. If we allow the possibility of intermanifold ("intersystem") crossing, the system at a given time (or on average) will have relative probabilities $p_{A}$ and $p_{B}$ of occupying these manifolds and will therefore exhibit a KS function

$$
F(E)=\ln \left[\frac{h^{3} c^{2}}{8 \pi E^{3}} \frac{p_{A} I_{A}(E)+p_{B} I_{B}(E)}{\sigma_{A}(E)+\sigma_{B}(E)}\right] .
$$


This is similar to Eq. (11) and has the same general behavior as that shown in Fig. 3 when the spectra are based on Gaussian profiles. In this case the probabilities, rather than the fixed concentrations, govern the spacing between the asymptotic lines. When intersystem crossing is sufficiently rapid, the probabilities attain the Boltzmann ratio and the lines merge, this being the limit of complete excited-state thermal equilibrium.

We have shown elsewhere $[37,40]$ how the intersystem crossing rate affects $T^{*}(E)$ under different assumptions in the case of Gaussian lines. Several rules of thumb have been developed: (a) The energy difference between the absorption peaks, in relation to their widths, determines whether the $T^{*}(E)$ feature is a peak or a divergence. (b) In the case of two bands of different strengths, the $T^{*}(E)$ feature, which is either a peak or a discontinuity, appears at an energy higher than the higher-energy absorption peak if that peak corresponds to the weaker transition; it falls at an energy lower than the lower-energy fluorescence peak if that peak corresponds to the weaker transition. (c) Varying the relative strength of the two transitions, or varying the concentration and strength jointly if a mixture is involved, changes the location of the $T^{*}(E)$ feature. (d) Changing the fluorescence yield of one component, or changing the intersystem crossing rate, changes the width and height of the $T^{*}(E)$ feature.

Any number $N$ of manifolds can be included in a picture like the one just developed for two manifolds. Unfortunately, the number of parameters escalates so fast that cases other than $N=2,3$ are not of great practical interest. The probabilities used in Eq. (13) may be either time-averaged or time-dependent, a possibility to which we will return in Sec. 6 .

\subsection{Single-manifold relaxation calculations}

The subject of excited-state relaxation is a huge one (see Mukamel [41]). Nonetheless, the simplicity of the $T^{*}(E)$ concept has prompted us to explore the subject with the help of some simple models. Work initiated by Sawicki (Rochester, unpublished) has shown that the computation of $T^{*}(E)$ by simple relaxation processes on a configuration-coordinate model is a highly problematic endeavor. In this model, Franck-Condon factors are employed to set up the initial occupation probabilities for excited-manifold vibrational states, after which these probabilities relax through a master equation with transition rates defined in a nearest-neighbor approximation. The emission is then computed, again using the set of Franck-Condon factors. The universal KS relation is reproduced for relaxation rates large compared with the radiative rate, but results for slower relaxation rates predict $T^{*}(E)$ features similar to nothing yet observed in any experiments we have analyzed. Also, a curious "crossing" theorem emerges, to the effect that $T^{*}\left(E_{0}\right)=T$ (ambient) for nearly any state of disequilibrium, where $E_{0}$ is the position that the emission peak would have if the system were equilibrated. Sawicki was able to demonstrate this both numerically and analytically on a single-configuration-coordinate model. No experiment, to our knowledge, either illustrates or confirms this theorem.

Research is in progress at the more complex reaches of relaxation theory, where Zhao (Rochester, unpublished) has also shown that the universal KS rẹ- 
lation formally emerges from the Brownian oscillator model [41] in the limit of ohmic dissipation. Zhao is developing a connection between this treatment and the Sawicki treatment.

\section{Application to chlorophyll $a$}

\subsection{Determination of $T^{*}(E)$ in various environments}

Spectral data from two laboratories based on differently-prepared chlorophyll a samples were used to obtain $T^{*}(E)$ in various solvents. A preliminary account of this work has been published [42] and we report here some of the first results of a kinetic analysis. Figure 6 shows the case of chlorophyll $a$ in acetone, measured in the two laboratories. The peaks in $T^{*}(E)$ occurring at $1.78 \mathrm{eV}$ are dissimilar in height and shape but we consider them to be real and equivalent. The reason for this confidence is that similar peaks - with similar variations in strength and shape - appear in the case of almost every chlorophyll $a$ environment analyzed. The case of acetonitrile solvent was published in our initial report [42]. The peak also occurs in the case of the peridinin-chlorophyll protein [43]. It is therefore reasonable to hypothesize that it is an intrinsic feature of the chlorophyll $a$ molecule itself.

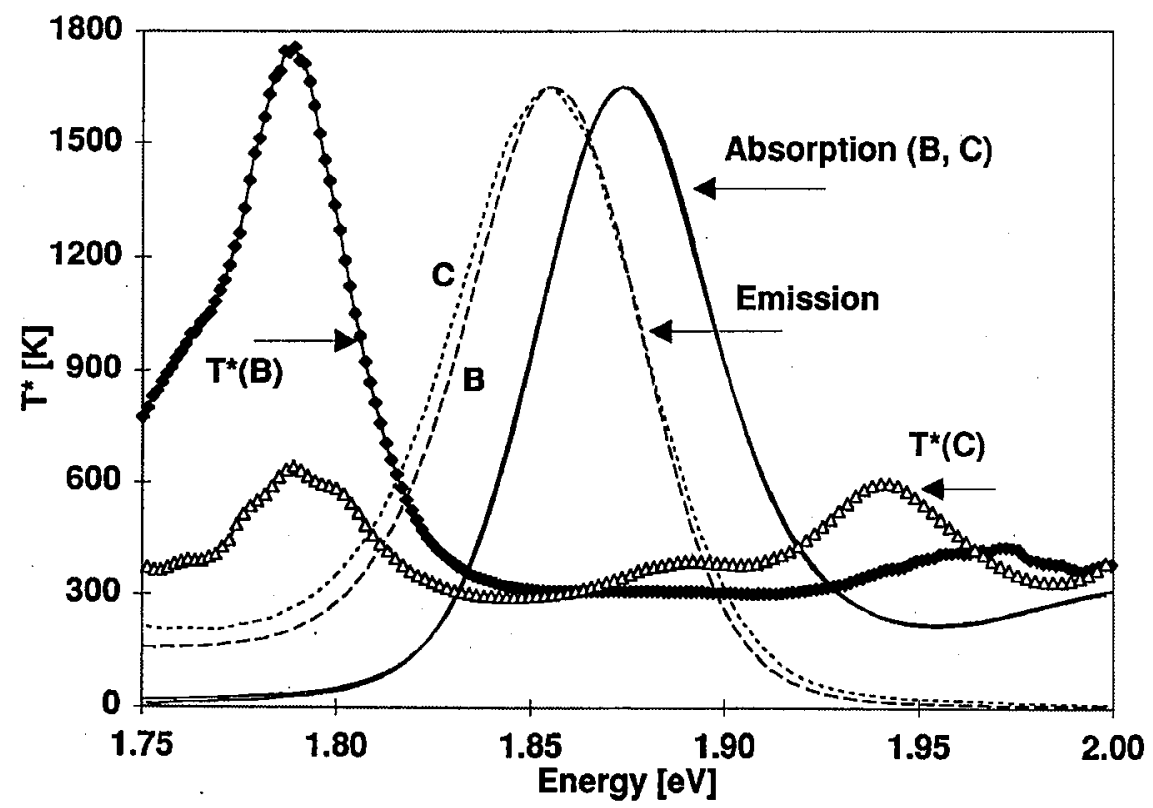

Fig. 6. Absorption, emission, and $T^{*}$ spectra of chlorophyll $a$ in acetone. $(B)$ Berkeley data by Talbot and $(C)$ Cornell data by Laible (see text). The peaks in $T^{*}$ near $1.78 \mathrm{eV}$ are apparently characteristic of the molecule. 


\subsection{Possible origin of the $T^{*}(E)$ peaks}

According to the rules of thumb given in Sec. 4.2, a peak in $T^{*}(E)$ lying to the red of the fluorescence band is likely to be caused by a weakly fluorescing manifold or manifolds at a lower energy than the main manifold. This has been borne out by fitting a kinetic model with two additional such manifolds, characterized by energies displaced from the main peak by $-50 \mathrm{meV}$ and $-100 \mathrm{meV}$. Two bands are needed for the best fit to the absorption and emission spectra, but an adequate fit can be obtained with one such state at $-50 \mathrm{meV}$. The rate of downward crossing to the new state is found, in all cases, to be within a factor of two of $0.06 \mathrm{~ns}^{-1}$. The oscillator strength of the new transition is of the order of $4 \%$ of that of the $Q_{y}$ transition.

It is suggested tentatively [44] that the new state is one of the excited triplet states of chlorophyll $a$, which are predicted theoretically to lie near the usual $S_{1}\left(Q_{y}\right)$ state. By two very different methods of calculation, triplet states are found to lie just below $S_{1}$ by $20 \mathrm{meV}$ or $73 \mathrm{meV}$ ( $T_{3}$ of Ref. [45] or $T_{2}$ of Ref. [46], respectively). It is reasonable that a triplet lying so much closer to the singlet would have an oscillator strength larger than the normal triplet $\left(T_{1}\right)$. The crossing rate we find is comparable to the usual intersystem $\left(S_{1} \rightarrow T_{1}\right)$ crossing rate, so the new level may act as an alternative pathway for entry into the triplet manifold. This model is currently under fuller analysis.

\subsection{Involvement of fluorescence yield in the KS relation}

In some early papers the fluorescence yield was included in the KS formalism by simply introducing it as a multiplicative factor with the fluorescence intensity. This device worked quite well in some instances [47], but is not an advisable procedure because the variable $E$ in the KS relation is tightly defined as the energy, or wavelength, of emission and absorption. The variable in the fluorescence yield is the excitation wavelength; it does not correct the emission at that wavelength. A consistent way to handle fluorescence yield is to make a parallel prediction of its excitation-wavelength dependence using the same kinetic scheme as the one that is used for $T^{*}(E)$, adjusting all parameters to fit both observables simultaneously. We are following this approach in our analysis of chlorophyll $a$, for which only sparse experimental fluorescence yield information is available [48].

\section{The case of time-resolved fluorescence}

If there is any question about whether observed spectra originate in equilibrated manifolds, there can hardly be any such question when the spectra are explicitly time-dependent. With some care the KS relation can be analyzed at each instant of time. In time-resolved fluorescence measurements, provided that the emission is primarily terminating in the ground state manifold, the derivation of Eq. (6) remains valid.

What might we expect of a time-resolved KS temperature set $T^{*}(E, t)$ ? For a cooling excited manifold in its simplest realization, a horizontal line $T^{*}(E, t)=$ $T^{\prime}(t)$ should fall toward an asymptotic horizontal line at ambient temperature. 


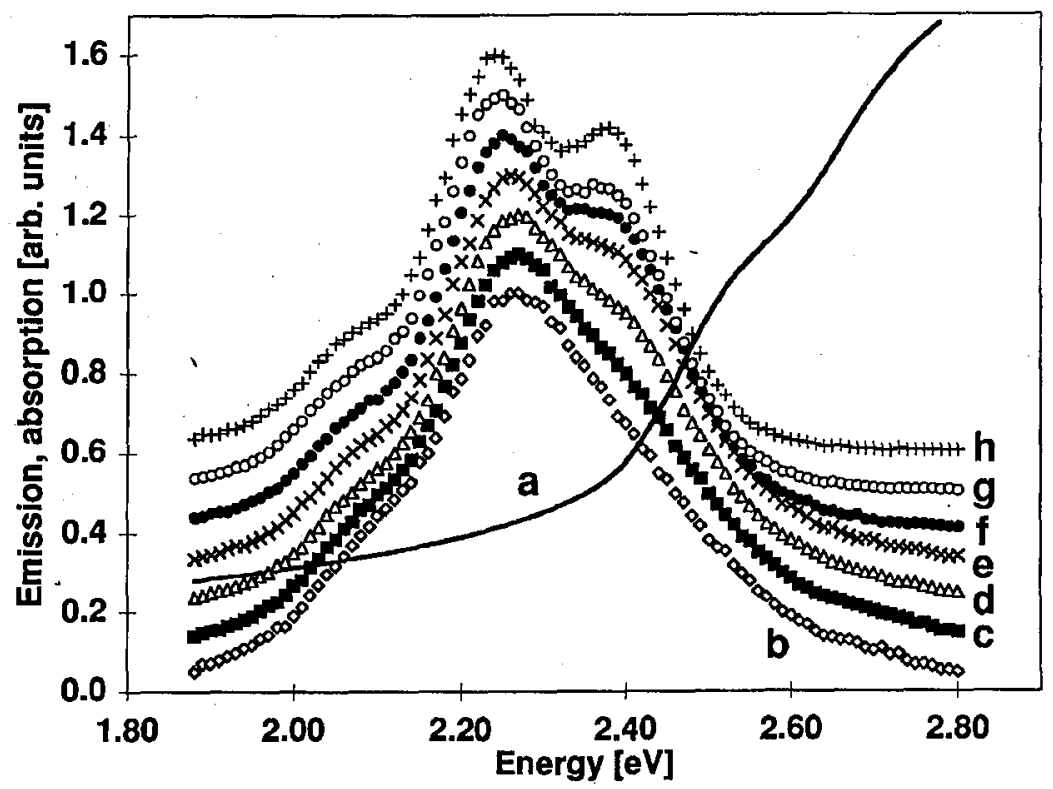

Fig. 7. Absorption and time-resolved fluorescence of PPV, poly(phenylenevinylene). Key: (a) absorption, (b) emission at $-100 \mathrm{fs}$, (c) $0 \mathrm{fs}$, (d) $100 \mathrm{fs}$, (e) $200 \mathrm{fs}$, (f) $500 \mathrm{fs}$, (g) $1000 \mathrm{fs}$, (h) $2000 \mathrm{fs}$, plotted from data by Kersting et al. [49]. For clarity, each of the spectra (c) $-(\mathrm{h})$ are displaced upward by 0.1 unit with respect to the next lower one. Fluorescence was measured by upconversion and the excitation pulse was $150 \mathrm{fs}$, centered at 0 fs.

More realistically, as noted in Sec. 2.2, the line should have some curvature as it falls. In the one experimental case analyzed to date, the result is quite surprising. Figure 7 shows time-resolved emission data from PPV [48] in which a shoulder develops into a peak on the high-energy side of the main emission band during the first $2 \mathrm{ps}$ after a $150 \mathrm{fs}$ pulse. The implied time-resolved $T^{*}(E, t)$, Fig. 8 , has two interesting features. At higher energies $(2.45-2.65 \mathrm{eV})$ there is a region that is essentially flat and falling, as if cooling were literally occurring. At low energies a strong peak is developing, remaining fixed at $2.35 \mathrm{eV}$ over the entire time course. In a sense we are seeing one of the steady-state $T^{*}(E)$ peaks actually being created.

\section{Summary and acknowledgments}

We have highlighted two approaches to the application of the KennardStepanov relation. Both begin with the construction of the function $F(E)$ from experimental emission and absorption spectra, using the first line of Eq. (7). In the standard approach, widely used since 1957, linear regions of $F(E)$ are selected or $F(E)$ as a whole is approximated by a linear function. Using the third line of Eq. (7), from the slope of the linear function a temperature is deduced as a single parameter $T^{*}$. Developing convincing explanations of this parametric $T^{*}$ has proved difficult. This may be partly due to the data having been collapsed into a single parameter; simplicity has a large price. If the data produce a parametric 


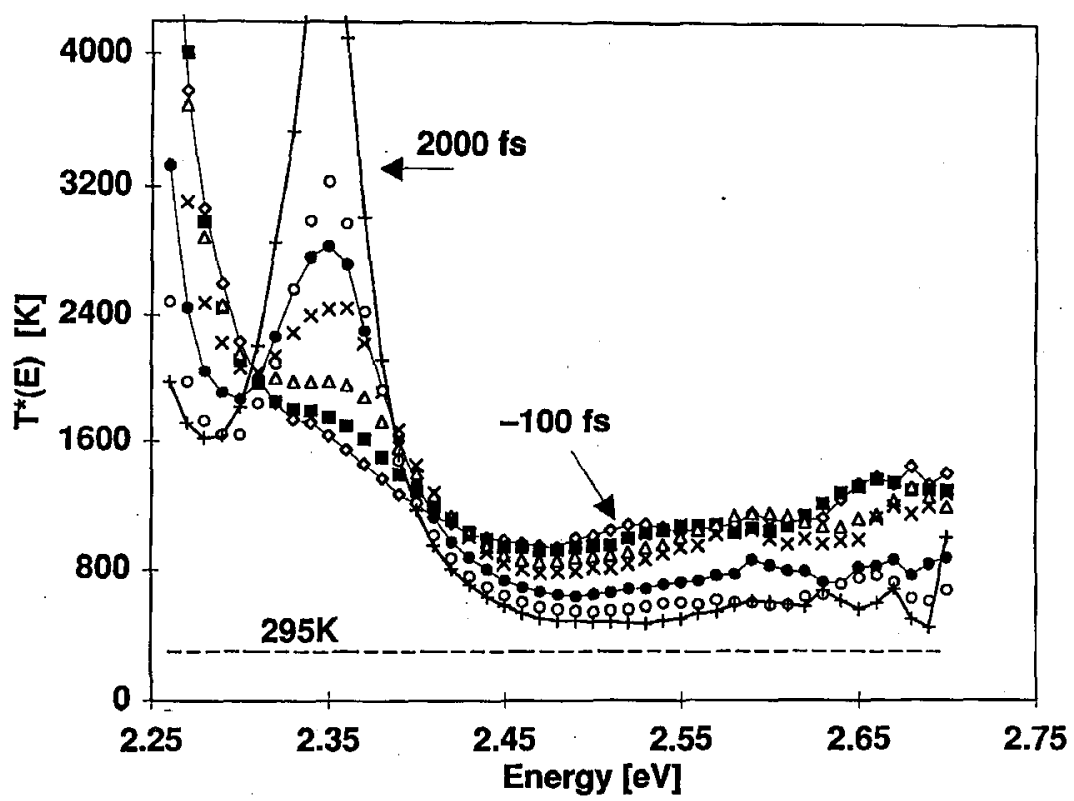

Fig. 8. $T^{*}(E)$ for PPV deduced from the spectra of Fig. 7. The charting symbols are the same as in Fig. 7, except that lines are drawn through the cases -100 fs, 500 fs and 2000 fs for guidance.

temperature significantly different from the ambient temperature $T$, it is clear that some premise of the KS relation has not been satisfied. The two major premises are thermal equilibration in the excited state and a homogeneous (or strongly energy-transferring) set of fluorescing entities.

The second approach to applying the relation [37] emphasizes the departure of $F(E)$ from linearity in $E$. The slope of $F(E)$ is evaluated at each $E$ and a temperature is defined, Eq. (12), producing a larger parameter set. This parameter set $T^{*}(E)$, which is not a function that is directly related mathematically to the system's spectra or its relaxation processes, has intriguing physical implications. We call it a "function" for convenience. In the Stokes region it frequently levels off and provides a reality check on the slopes deduced by the first approach. Aside from demonstrating in greater detail whether or not the equilibration criterion is met, $T^{*}(E)$ displays features that have been related to very elementary relaxation processes.

Is $T^{*}(E)$ a large advance over the single parameter $T^{*}$ ? In all candor we admit no greater success in finding detailed explanations for most of the $T^{*}(E)$ sets we have analyzed, but the intrigue persists. This is nowhere better appreciated than in the time-resolved case. The ultimate value of the alternative KS analysis rests on the degree of success that may be attained in the interpretation of $T^{*}(E)$ and $T^{*}(E, t)$. Primarily, a connection must be made between $T^{*}(E, t)$ and modern theories of excited-state relaxation. By definition, this is tantamount to predicting time-resolved spectra with a consistent set of parameters for any given system and 
the formalism for such an effort exists [41]. The prospect held out by $T^{*}(E)$ is the possible identification of relaxation parameters or processes that are determined ambiguously by the spectra alone, or that are obscured by observed spectral features not directly affected by relaxation. The coupled-two-KS-manifold model of Sec. 2 is a very elementary example of these possibilities.

The author thanks the many scientists, particularly Drs. Harald Kauffmann, Philip Laible, and Mary Talbot, who have transmitted their emission and absorption data sets. He also thanks Philip Laible, Thomas Owens, and Kenneth Sauer for frequent helpful discussions of the KS theory and related matters. Assistance in preparation of the manuscript by Jamin Brown and Aleksander Radunskiy was greatly appreciated. The research was supported in part by the U.S. Department of Agriculture NRICGO grant 95-37306-2014 and in part by National Science Foundation grant PHY-94-15583.

\section{References}

[1] E. Pringsheim, Physik Z. 14, 129 (1913).

[2] E.H. Kennard, Phys. Rev. 11, 29 (1918).

[3] E.H. Kennard, Phys. Rev. 28, 672 (1926).

[4] B.I. Stepanov, Dokl. Akad. Nauk SSSR 112, 839 (1957) [Sov. Phys. Dokl. 2, 81 (1957)].

[5] B.S. Neporent, Dokl. Akad. Nauk SSSR 119, 682 (1958) [Sov. Phys. Dokl. 3, 337 (1958)].

[6] S. Mukamel, V. Rupasov, Chem. Phys. Lett. 242, 17 (1995).

[7] P.D. Laible, R.S. Knox, T.G. Owens, J. Phys. Chem. B 102, 1641 (1998).

[8] F. Perrin, Ann. Phys. (Paris) 12, 169 (1929).

[9] A. Jabłoński, Z. Phys. 96, 236 (1935).

[10] P. Pringsheim, Z. Phys. 57, 739 (1929).

[11] A. Jabłoński, Nature 131, 839 (1933).

[12] P. Pringsheim, J. Phys. USSR 10, 495 (1946).

[13] S.I. Vavilov, J. Phys. USSR 10, 499 (1946).

[14] D.E. McCumber, Phys. Rev. 136, A954 (1964).

[15] Y.B. Band, D.F. Heller, Phys. Rev. A 38, 1885 (1988).

[16] I. Ketskemety, L. Szalay, Z. Varkonyi, Acta Phys. Chem. (Szeged) 11, 15 (1965).

[17] L.O. Björn, G.S. Björn, Photochem. Photobiol. 44, 535 (1986).

[18] A.V. Altaiskaya, N.G. Bakhshiev, I.V. Piterskaya, Opt. Spektrosk. 27, 1013 (1969) [Opt. Spectrosc. 27, 550 (1969)].

[19] G.S. Singhal, J. Hevesi, Photochem. Photobiol. 14, 509 (1971).

[20] A. Kawski, J. Kukielski, Z. Naturforschung 25a, 653 (1970).

[21] J. Hevesi, L. Kozma, Acta Phys. Chim. (Szeged) 8, 103 (1962).

[22] N.R. Senatorova, L.V. Levshin, B.D. Ryzhikov, Opt. Spektrosk. 50, 574 (1981) [Opt. Spectrosc. 50, 311 (1981)].

[23] E.A. Andreeshchev, V.S. Viktorova, S.F. Kilin, Yu.P. Kushakevich, I.M. Rozman, Opt. Spektrosk. 24, 723 (1968) [Opt. Spectrosc. 24, 387 (1968)]. 
[24] A. Kawski, J. Kukielski, J. Lumin. 4, 155 (1971).

[25] N.A. Borisevich, V.V. Gruzinskii, Opt. Spektrosk. 14, 39 (1963) [Opt. Spectrosc. 14, $20(1963)]$.

[26] A. Balter, J. Lumin. 26, 99 (1981).

[27] J. Hevesi, L. Kozma, L. Szalay, Acta Phys. Pol. 29, 57 (1966).

[28] A. Jabłoński, Acta Phys. Pol. 26, 427 (1964); Bull. Acad. Sci. Pol. 20, 243 (1972).

[29] R.L. Van Metter, R.S. Knox, Chem. Phys. 12, 333 (1976).

[30] J. Hevesi, G.S. Singhal, Spectrochim. Acta A 25, 1751 (1969).

[31] A.V. Sechkarev, V.N. Beger, Opt. Spektrosk. 72, 560 (1992) [Opt. Spectrosc. 72, 303 (1992)].

[32] V.V. Gruzinskii, N.A. Borisevich, Opt. Spektrosk. 15, 457 (1963) [Opt. Spectrosc. 15, 246 (1963)].

[33] N.G. Bakhshiev, Yu.T. Mazurenko, I.V. Piterskaya, Opt. Spektrosk. 21, 550 (1966) [Opt. Spectrosc. 21, 307 (1966)]; Yu.T. Mazurenko, N.G. Bakhshiev, Opt. Spektrosk. 28, 905 (1970) [Opt. Spectrosc. 28, 490 (1970)].

[34] R.L. Van Metter, Ph.D. thesis, University of Rochester, Rochester 1977, p. 18.

[35] L.O. Björn, G.S. Björn, Photochem. Photobiol. 44, 535 (1986).

[36] K. Sauer, private communication reported in Ref. [37]; S.C. Switalski, K. Sauer, Photochem. Photobiol. 40, 423 (1984). See the discussion of the data in Ref. [37].

[37] D.A. Sawicki, R.S. Knox, Phys. Rev. A 54, 4837 (1996).

[38] P.D. Laible, Ph.D. thesis, Cornell University, Ithaca 1995.

[39] M.R. Robinson, H. Rasafitrimo, Y. Gao, B.R. Hsieh, Polym. Mater. Sci. Eng. 74, 292 (1996).

[40] R.S. Knox, Pure Appl. Chem. 69, 1163 (1997).

[41] S. Mukamel, Principles of Nonlinear Optical Spectroscopy, Oxford University Press, New York 1995, especially Chaps. 8 and 9.

[42] R.S. Knox, P.D. Laible, D.A. Sawicki, M.F.J. Talbot, J. Lumin. 72-74, 580 (1997).

[43] S. Akimoto, S. Takaichi, T. Ogata, Y. Nishimura, I. Yamazaki, M. Mimuro, Chem. Phys. Lett. 260, 147 (1996); F.J. Kleima, personal communication (1998). Data files were kindly supplied by Prof. Mimuro (samples by Dr. Ogata, from Alexandrium cohorticula), and Dr. Kleima (samples from Amphidinium carterae).

[44] R.S. Knox, J.S. Brown, P.D. Laible, M.F.J. Talbot, in preparation.

[45] J.C. Chang, Ph.D. thesis, University of Rochester, Rochester, NY, 1972, p. 104.

[46] J.D. Petke, G.M. Maggiora, L.L. Shipman, R.E. Christofferson, Photochem. Photobiol. 30, 203 (1979).

[47] I. Ketskemety, J. Dombi, R. Horvai, Ann. Phys. (Leipzig) [7] 8, 342 (1961).

[48] D. Frąckowiak, Bull. Acad. Sci. Pol. Ser. Math. Astron. Phys. 11, 561 (1963).

[49] R. Kersting, B. Mollay, M. Rusch, J. Wenisch, G. Leising, H.F. Kauffmann, J. Chem. Phys. 106, 2850 (1997). 